\title{
A Mathematical Model for Location Transformation Based on Light and Shadow Length
}

\author{
Zhang Lu-ping \\ JiangXi University of Technology ,Institute of Science
}

\begin{abstract}
The shadow direction as well as length of objects will change along with the change of sun shinning over the earth and this paper establishes a model to research on the shadow changes in a certain period with a same pole in the same position so as to know the time changes and location.
\end{abstract}

Key Words- Sun elevation angle; Longitude; Latitude

\section{INTRODUCTION}

When the sun shines over opaque objects, black shadows shall be generated on the ground and suppose the observation point does not change, the shadow of objects shall change along with the time changes because of the sun. This paper constitutes by three parts. The first part determines the observation time, location so as to study on the change of shadow length; the second part determines the observation location based on the observation time and shadow vertex; finally, by analyzing the shadow length changes based on time to determine the concrete location of objects and observation time.

\section{ChangeS OF LENGTH OF SHADOW}

Take the contact point of pole and ground as the initial point and the distance between the initial point to end of pole shadow is known as the length of pole shadow which changes along with earth revolution and rotation which can be calculated based on the sun elevation angle. The earth revolution effect is depicted based on declination of sun and geographic latitude, $\varphi$ represents declination of sun while $\beta$ stands for geographic latitude (both of them have negative as well as positive exponent and the positive angle is northern latitude while the negative angle is southern latitude). What's more, the hour angle is $t$ and $T$ refers to the local time in 24-hour format, sun elevation angle demonstrate in $\alpha_{\text {[1], then }}$

$$
\sin \alpha=\sin \beta \sin \varphi+\cos \beta \cos \varphi \cos t ;
$$

The fixed location and pole do not change and the effect of rotation mainly shows in the changes of hour angle $t$. A time lapse of rotation is $360^{\circ}$ and 24 hours for one day $\&$ night then every hour the change is

Then the computing formula for shadow length a is:

$$
a=l \cot [\arcsin (\sin \beta \sin \phi+\cos \beta \cos \phi \cos t)] 。
$$

Based on the above computing formula for shadow length a, we can have a good knowledge of the changes of shadow

(1)The pole shadow length $a$ is proportional to the height $l$.

(2)The pole shadow length $a$ is inverse to sun elevation angle $\alpha$.

(3)While pole shadow length $a$ and hour angel stand at $\left[-90^{\circ} \sim 0^{\circ}\right]$, namely from 6:00 to $12: 00$ of $15^{\circ}$. The approximate change of pole shadow for one day \& night is: the shadow length in sunrise and sunset is the longest and shortest in high noon. Therefore, the high noon hour angle is beckoned as 0 ,

$t=15^{\circ} \times(T-12) 。$
Beijing time, there shall be monotone decreasing; if stand at $\left[0^{\circ} \sim 90^{\circ}\right]$, namely from 12:00 to $18: 00$, there shall be monotonically increasing.

The observation time is from 9:00 to 15:00 Beijing time on 22nd October 2015 in Tian'anmen Square (northern latitude $39^{\circ} 54^{\prime} 26^{\prime \prime}$ and east longitude $116^{\circ} 23^{\prime} 29^{\prime \prime}$ ), namely the solar declination 
should be ${ }^{\varphi}=10.83^{\circ}$, geographic latitude should be

$t \in\left[-45^{\circ} \sim 45^{\circ}\right]$ and $l=3$ meters as example: $\beta=39.91^{\circ}$. Take the data on the condition that

\begin{tabular}{|c|c|c|c|c|c|c|c|c|}
\hline Time & $9: 00$ & $9: 04$ & $9: 08$ & $9: 12$ & $9: 16$ & $9: 20$ & $9: 24$ & $9: 28$ \\
\hline Length (m) & 7.36 & 7.14 & 6.94 & 6.74 & 6.56 & 6.39 & 6.23 & 6.08 \\
\hline Time & $9: 32$ & $9: 36$ & $9: 40$ & $9: 44$ & $9: 48$ & $9: 52$ & $9: 56$ & \\
\hline Length (m) & 5.93 & 5.79 & 5.66 & 5.54 & 5.42 & 5.31 & 5.20 & \\
\hline Time & $10: 00$ & $10: 04$ & $10: 08$ & $10: 12$ & $10: 16$ & $10: 20$ & $10: 24$ & $10: 28$ \\
\hline Length (m) & 5.10 & 5.01 & 4.91 & 4.83 & 4.74 & 4.67 & 4.59 & 4.52 \\
\hline Time & $10: 32$ & $10: 36$ & $10: 40$ & $10: 44$ & $10: 48$ & $10: 52$ & $10: 56$ & \\
\hline Length (m) & 4.45 & 4.39 & 4.33 & 4.27 & 4.21 & 4.16 & 4.11 & \\
\hline Time & $11: 00$ & $11: 04$ & $11: 08$ & $11: 12$ & $11: 16$ & $11: 20$ & $11: 24$ & $11: 28$ \\
\hline Length (m) & 4.07 & 4.03 & 3.99 & 3.95 & 3.91 & 3.88 & 3.85 & 3.82 \\
\hline Time & $11: 32$ & $11: 36$ & $11: 40$ & $11: 44$ & $11: 48$ & $11: 52$ & $11: 56$ & \\
\hline Length (m) & 3.80 & 3.77 & 3.75 & 3.74 & 3.72 & 3.71 & 3.69 & \\
\hline Time & $12: 00$ & $12: 04$ & $12: 08$ & $12: 12$ & $12: 16$ & $12: 20$ & $12: 24$ & $12: 28$ \\
\hline Length (m) & 3.69 & 3.68 & 3.67 & 3.67 & 3.67 & 3.67 & 3.68 & 3.68 \\
\hline Time & $12: 32$ & $12: 36$ & $12: 40$ & $12: 44$ & $12: 48$ & $12: 52$ & $12: 56$ & \\
\hline Length (m) & 3.69 & 3.70 & 3.72 & 3.73 & 3.75 & 3.77 & 3.79 & \\
\hline Time & $13: 00$ & $13: 04$ & $13: 08$ & $13: 12$ & $13: 16$ & $13: 20$ & $13: 24$ & $13: 28$ \\
\hline Length (m) & 3.82 & 3.84 & 3.87 & 3.91 & 3.94 & 3.98 & 4.02 & 4.06 \\
\hline Time & $13: 32$ & $13: 36$ & $13: 40$ & $13: 44$ & $13: 48$ & $13: 52$ & $13: 56$ & \\
\hline Length (m) & 4.10 & 4.15 & 4.20 & 4.26 & 4.31 & 4.37 & 4.44 & \\
\hline Time & $14: 00$ & $14: 04$ & $14: 08$ & $14: 12$ & $14: 16$ & $14: 20$ & $14: 24$ & $14: 28$ \\
\hline Length (m) & 4.50 & 4.57 & 4.65 & 4.73 & 4.81 & 4.89 & 4.99 & 5.08 \\
\hline Time & $14: 32$ & $14: 36$ & $14: 40$ & $14: 44$ & $14: 48$ & $14: 52$ & $14: 56$ & $15: 00$ \\
\hline Length (m) & 5.18 & 5.29 & 5.40 & 5.51 & 5.64 & 5.76 & 5.90 & 6.04 \\
\hline
\end{tabular}


While adopting MATLAB software programming[2], we are able to draw the changing curve of a three-meter high pole shadow length.

$$
a=\sqrt{x^{2}+y^{2}}
$$

III. DETERMINE THE POLE LOCATION BASED ON THE END OF POLE SHADOW

$x$ refers to the abscissa of the end of pole shadow while $y$ refers to the ordinates of the end of pole shadow and $a$ refers to the shadow length. Then, we can have

The following data

\begin{tabular}{|c|c|c|c|c|}
\hline Beijing Time & $\begin{array}{c}\text { Abscissa } \\
\text { (Unit: meter) }\end{array}$ & $\begin{array}{c}\text { ordinates } \\
\text { (Unit: meter) }\end{array}$ & $\begin{array}{c}\text { Hour angle } \\
\text { (Unit: meter) }\end{array}$ & $\begin{array}{l}\text { Shadow length } \\
\text { (Unit: meter) }\end{array}$ \\
\hline $14: 42$ & 1.0365 & 0.4973 & 40.5 & 1.149626 \\
\hline $14: 45$ & 1.0699 & 0.5029 & 41.25 & 1.182199 \\
\hline $14: 48$ & 1.1038 & 0.5085 & 42 & 1.215297 \\
\hline $14: 51$ & 1.1383 & 0.5142 & 42.75 & 1.249051 \\
\hline $14: 54$ & 1.1732 & 0.5198 & 43.5 & 1.283195 \\
\hline $14: 57$ & 1.2087 & 0.5255 & 44.25 & 1.317993 \\
\hline $15: 00$ & 1.2448 & 0.5311 & 45 & 1.353364 \\
\hline $15: 03$ & 1.2815 & 0.5368 & 45.75 & 1.389387 \\
\hline $15: 06$ & 1.3189 & 0.5426 & 46.5 & 1.426153 \\
\hline $15: 09$ & 1.3568 & 0.5483 & 47.25 & 1.4634 \\
\hline $15: 12$ & 1.3955 & 0.5541 & 48 & 1.501482 \\
\hline $15: 15$ & 1.4349 & 0.5598 & 48.75 & 1.540232 \\
\hline $15: 18$ & 1.4751 & 0.5657 & 49.5 & 1.579853 \\
\hline $15: 21$ & 1.516 & 0.5715 & 50.25 & 1.620145 \\
\hline $15: 24$ & 1.5577 & 0.5774 & 51 & 1.661271 \\
\hline $15: 27$ & 1.6003 & 0.5833 & 51.75 & 1.703291 \\
\hline $15: 30$ & 1.6438 & 0.5892 & 52.5 & 1.746206 \\
\hline $15: 33$ & 1.6882 & 0.5952 & 53.25 & 1.790051 \\
\hline $15: 36$ & 1.7337 & 0.6013 & 54 & 1.835014 \\
\hline $15: 39$ & 1.7801 & 0.6074 & 54.75 & 1.880875 \\
\hline $15: 42$ & 1.8277 & 0.6135 & 55.5 & 1.927918 \\
\hline
\end{tabular}

\section{DETERMINE THE LONGITUDE OF POLE}

MATLAB software can be adopted to import vertex coordinate $(x, y)$ of the given shadow end, and hour angle $t_{\text {so }}$ as to carry out secondary curve-fitting which is able to have a general expression as well as fitting image of a quadratic function in terms of shadow length $a$ and hour angle $t$. At last, the lowest abscissa can be obtained, which is the deviation degree of noon time 12:00, and the longitude deviates from $120^{\circ}$ east longitude, showing with $\lambda$. After observing the geographic longitude $\theta$, we can have:

$\theta=120^{\circ} \pm \lambda$ 
Then, all coordinate data of given shadow vertex and obtained hour angle $t$ can be imported to MATLA to carry out secondary curve-fitting and we can have the a general expression as well as fitting image of a quadratic

$$
a=0.00066179 t^{2}-0.0118809 t+0.5469176
$$

According to the general expression of this quadratic function in terms of shadow length $a$ and hour angle $t$ and the general expression of quadratic function shows as follows:

function we can have the lowest abscissa showing as $\lambda$

which derives from $120^{\circ}$ east longitude. Namely,

$$
\lambda=\frac{0.0118809}{2 \times 0.00066179}=8.976472^{\circ}
$$

The geographic longitude of observation location is:

$$
\theta=120^{\circ}-\lambda=120^{\circ}-8.976472^{\circ}=111.023528^{\circ}
$$

Namely, the geographic longitude of observation

Based on the responding change of (3), we can have

location $\theta$ is $111^{\circ} 1$ ' 25 ',

the following expression demonstrating pole length $l$ :

Determine the latitude of pole

$$
l=a \tan [\arcsin (\sin \beta \sin \varphi+\cos \beta \cos \varphi \cos t)]
$$

This paper constructs a model showing the concrete changes of pole length $l$ and geographic latitude $\beta$ of observation location while taking hour angle $t$ and shadow length $a$ as variables. Then, the hour angle $t$, shadow length $a$ and solar declination $\varphi$ can be substituted into formula (5). What's more, by adopting MATLAB software programming we can carry out nonlinearity curve-fitting, which can estimate the approximate range of pole length $l$ and geographic latitude $\beta$ of observation location

The time is able to determine the solar declination $\varphi=10.6167^{\circ}$ software programming, curve-fitting so as to have the approximate value of pole length $l$ and geographic latitude $\beta$ of observation location, $l \approx 1.8940$ and $\beta \approx 17.4462^{\circ}$. To sum up, the geographic longitude of observation location is $111^{\circ} 1$ '25' ' and $17^{\circ} 26^{\prime} 46^{\prime}$ ' for geographic latitude $\beta$. In addition, through the latitude and longitude positioning website, we input $\theta=111^{\circ} 1^{\prime} 25^{\prime \prime}$ and $\beta=17^{\circ} 26^{\prime} 46^{\prime \prime}$ to clearly position the location, which is near the Xisha Islands.

\section{MODEL PROMOTION}

By shooting object video we can also determine the object location and recording date, which mainly adopts Total Video Converter to cut the image and then analyze these images to have the coordinate of pole shadow vertex and shadow length. Concrete operations show as follows: we cut one image per two minutes however, the picture is too large in original video and we only need the images for shadow changing. Before cutting images, we firstly cut frame size to remove unnecessary parts and set custom options to choose each image per three seconds to have 7,324 pictures. Among which, we choose one among 360 pictures (namely per two minutes) to have 20 pictures. In addition, the incidental imread function of MATLAB can help to read these 20 pictures and demonstrate in the form of formats and we can have 20 tables xi. As time changes, the location of shadow changes while coordinate value also changes. While subtract the close two tables we can have table yi and then we get the sum to have a new table which is three-dimensional data. Then, with the help of rgb2gray function and imadjust function we can transform the three-dimensional table to two-dimensional table. At last, imshow order can be adopted to restore the image to have the changing trajectory of shadow. These 20 pictures cannot help to directly see the change of shadows which have to be processed, to be matriculated and digitized. Each point in the picture has a responding elementary data and while we open the program to enlarge for 800 times and meanwhile point at shadow 
vertex we can have responding coordinates which are beneficial to have the coordinates of pole. Suppose the bottom is the initial point and we subtract these two, we can have relative coordinate of shadow vertex. Finally, taking advantage of the formula 1 and 2, we are able to approximately determine the geographic longitude, geographic latitude of observation location, pole length as well as observation date.

\section{ACKNOWLEDGEMENTS}

This work was supported by Probability and Mathematical Statistics Distinguished Courses of JiangXi University of Technology, Project Number KC1402

\section{REFERENCES}

[1] Wang Guoan, Mi Hongtao, Deng Tianhong, Li Yanan, Li Lanxia. Variation change calculation on sun elevation angle and solar azimuth in sunrise and sunset for one year. METEOROLOGICAL AND ENVIRONMENTAL SCIENCES, 2007, B09 issue: 161-164.

[2] Zhou Pin. MATLAB Probability Theory and Mathematical statistics. Tsinghua University Press, 2012 : 54-59.

[3] Yang Zeying. Discussion on the object shadow. Periodical Scientific Inquiry, 2012, 11th issue: 186-186.

[4] Pan Junchun. An excellent Video Format Conversion Tools: E.M. Total Video Converter, China Information Technology Education, 2015, 12th issue: 61-62. 